\title{
Modified GPSR Protocol for Wireless Sensor Networks
}

\author{
L. Nithyanandan, G. Sivarajesh and P. Dananjayan
}

\begin{abstract}
Greedy Perimeter Stateless Routing (GPSR) is a proven efficient geographical routing protocol for wireless adhoc network which is symmetric in nature. It also offers routing support for Wireless Sensor Network (WSN). However, GPSR was designed for the symmetric links (bidirectional reachable), but sensor networks are often asymmetric in nature. Moreover in WSN the nodes are not designated through their IP addresses instead they are marked with their locations. So, when the destination is outside the boundary GPSR suffers by energy inefficiency as it has to trace through all the nodes in the boundary for reaching the destination. In addition to this, another key problem is data inconsistency which becomes a major challenge in deploying GPSR in WSN. In this paper a modified version of GPSR is proposed which identifies optimal route based on energy utilization and overcome problems in GPSR so that the feasibility of using GPSR in asymmetric WSN can be increased. The simulation results prove that the energy and delay is minimized and hence the proposed protocol outperforms the existing routing protocol for WSN.
\end{abstract}

Index terms - Wireless sensor networks, GPSR, Ad hoc, Routing protocol

\section{INTRODUCTION}

A Wireless Sensor Network (WSN) is a network consisting of individual nodes that are spatially distributed devices using sensors to cooperatively monitor physical or environmental conditions at different locations as shown in Fig.1. These nodes have to collaborate in order to fulfill their tasks and wireless communication is used to enable this collaboration [1]. WSN nodes are battery powered, the routing protocol should consume less energy and also it should be ensured that the information transfer delay should be less. Various routing protocols are available for wireless networks. Most of the routing protocols that were studied are unsuitable for the use in WSN or had issues with implementation on highly resource-constrained nodes.

Dynamic Source Routing (DSR) and Ad-hoc On-demand Distance Vector (AODV) are arguably the most popular of all routing protocols [2,3]. However, due to their extensive use of routing tables and source routing, they render

Paper submitted on 20.08.2009.

L.Nithyanandan is with the Department of Electronics and Communication Engineering, Pondicherry Engineering College as Assistant Professor. (email:nithi@pec.edu)

G.Sivarajesh was with the Department of Electronics and Communication Engineering, Pondicherry Engineering College as P.G.student.

P.Dananjayan is with the Department of Electronics and Communication Engineering, Pondicherry Engineering College as Professor. (email:pdananjayan@rediffmail.com)1

2 Department of Electronics and Communication Engineering,

Pondicherry Engineering College, Pondicherry. themselves inappropriate for routing in WSN. Location Aided Routing (LAR) introduces the concept of routing using geographical positions of nodes [4]. It also demonstrates the use of smart flooding to build the routes. Nevertheless, it is still the source routing protocol for adhoc networks. Low Energy Adaptive Clustering Hierarchy (LEACH) is the first routing protocol for WSN that was studied [5]. However, LEACH is unsuitable for use in most environments that do not involve base station routing or data aggregation. Conversely, Delay-Energy Aware Routing Protocol (DEAP) has an adaptive energy management scheme [6] where the load is being shared to all neighbouring nodes. Therefore the time consumed to wake all neighbouring nodes would be considered which in turn increases packet delivery time. However, delay-aware robust forwarding (DWARF) has reduced its delay time due to the flooding technique used in this routing environment [7]. But the optimization of cluster in this routing is not an efficient one which would consume more energy. Greedy Perimeter Stateless Routing (GPSR) protocol makes use of the IEEE 802.11 MAC layer [8] and suits well for symmetric adhoc networks. However, none of these protocols support an optimal mode for delivering messages efficiently in a WSN. They do not provide any service for distinguishing the importance of delivering messages in WSN. Hence any one of the routing technique that adapts well for the WSN should be analyzed and modified so as to suit the requirement of WSN networks. In this line it is found GPSR happens to be a better choice and a modified GPSR is being proposed in this paper and proved to support for delivering messages efficiently and effectively in WSN.

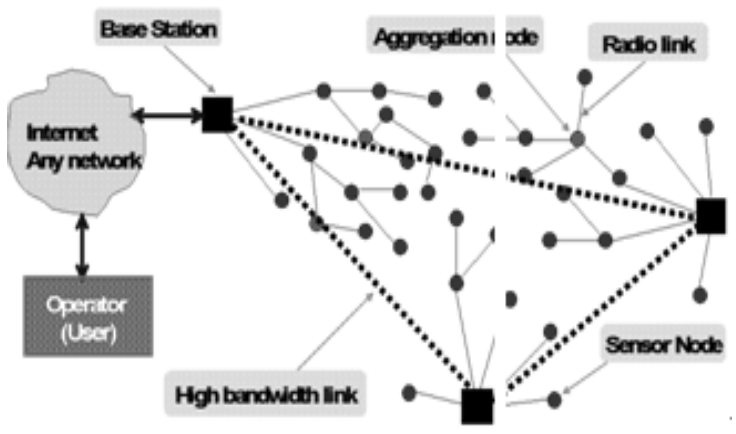

Fig. 1 Basic architecture of sensor network

\section{GPSR}

GPSR protocol [8] is the earliest geographical routing protocols for adhoc networks which can also be used for WSN environment. The GPSR adapts a greedy forwarding strategy and perimeter forwarding strategy to route messages. It makes uses of a neighbourhood beacon that sends a node's identity and its position. However, instead of 
sending this beacon periodically and add to the network congestion, GPSR piggybacks the neighbourhood beacon on every message that is sent or forwarded by the node. Every node in GPSR has a neighbourhood table of its own. Whenever a message needs to be sent, the GPSR tries to find a node that is closer to the destination than itself and forwards the message to that node. However, this method fails for topologies that do not have a uniform distribution of nodes or contain voids. Hence, the GPSR adapts to this situation by introducing the concept of perimeter routing utilizing the right-hand graph traversal rule. Every packet transmitted in GPSR has a fixed number of retransmits [1, 8]. This information is given to the node by the medium access (MAC) layer that is required to be compliant to the IEEE 802.11 standard. This may render the GPSR protocol unusable in its normal form for WSN. The GPSR does not elucidate more on the action taken in case a message is unable to be transmitted even in perimeter mode. Finally GPSR disallows the use of periodic broadcast of the neighbourhood beacons and piggybacks these beacons on the messages sent by each node. As a strong geographical routing protocol GPSR is allowing nodes to send packets to a particular location and holding a promise in providing routing support in WSN. Many recent research works in WSN are building applications using GPSR protocol. However, GPSR is not originally designed for sensor networks, several problems are required to be fixed before it is applied in sensor networks.

First, GPSR is designed under the assumption of symmetric wireless links. That is, whenever a node receives a beacon from another node, it considers that node as its neighbour and they are bidirectional reachable. Such an assumption may not be realistic for practical sensor networks, since wireless links in sensor networks often are asymmetric. In sensor networks, packet destinations are often marked with locations instead of identifiers like IP addresses and packets finally reach the node geographically closest to the destination, the home node of the target location. Because sensor nodes may become irregular after running for a period of time or due to unattended deployment, it is highly possible that the target location in a packet is located outside the exterior perimeter of the sensor network. In such cases, GPSR's planar perimeters algorithm does not work efficiently and all such packets have to visit all nodes on the border of the network topology before returning and recognizing the home node. This process is very energy expensive. Therefore, the concept aggregation node is introduced where energy is efficiently used. Data consistency problem, which means data retrieved from a location in sensor networks should be consistent with data sent to the other location, becomes a challenge due to the dynamic nature of sensor networks [9]. Sensor nodes including the current home nodes for any locations may often fall into disfunction state or even die due to hardware failure and energy exhaustion or intermittently reachable, as a result of the impact of various factors, e.g., environmental effects. Improvement in system lifetime increases as the ratio of idle-to-sleep energy consumption increases and as the density of the network increases. Each node needs to awake at a fixed time frame to make the delivery of the data successful. High node density results in existence of several paths between two given nodes whose path length and delay characteristics are similar. Hence the selection of path needs to be enhanced to analyze proper routing technique with optimization in all aspects. The main objective of the paper is to propose a routing protocol for WSN so as to ensure successful data delivery, less packet delay and optimal energy consumption by modifying GPSR.

\section{MODIFIED GPSR}

The proposed routing scheme is based on the fact that the energy consumed to send a message to a distant node is greater than the energy needed for a short range transmission. GPSR protocol is extended using aggregation node or head set node. Aggregation node is responsible for transmitting messages to the distant base station and routing is decided using the respective head set members. The head set is decided on a routine basis with reference to the energy level of the signal received to the base station at the time of reception of "hello packets". At one time, only one member of the head set is active and the remaining head set members are in sleep mode. The task of transmission to the base station is uniformly distributed among all the head set members similar to LEACH protocol. Each cluster has a head set that consists of several virtual cluster heads. The operation on this network involves two process, selection of head sets for the clusters and members of head set transmits data to the base station. Each member of a head set becomes a cluster head once during an epoch depending on their battery power level. At the start, a set of cluster heads are chosen on random basis. These cluster heads send a short range advertisement broadcast message. The sensor nodes receive the advertisements and choose their cluster heads based on the signal strengths of the advertisement messages. Each sensor node sends an acknowledgment message to its cluster head. Moreover, the cluster heads choose a set of associates based on the signal analysis of the acknowledgments. A head set consists of a cluster head and the associates. The head set, which is responsible to send messages to the base station, is chosen for each time based on the energy level of the signal received to the base station. The non-cluster head nodes collect the sensor data and transmit the data to the cluster head, in their allotted time slots. The cluster head node must keep its radio turned on to receive the data from the nodes in the cluster. The associate members of the head set remain in the sleep mode and do not receive any messages. After, some pre-determined time interval, the next associate becomes a cluster head and the current cluster head becomes a passive head set member.

For a sensor network of $\mathrm{n}$ nodes, the optimal number of clusters is given as $\mathrm{k}$. All nodes are assumed to be at the same energy level at the beginning. The amount of consumed energy is same for all the clusters. At the start of the election phase, the base station randomly selects a given number of cluster heads. The cluster heads broadcast messages to all the sensors in their neighbourhood $[10,11]$. Then the sensors receive messages from one or more cluster heads and choose their cluster head using the received signal strength. Later, the sensors transmit their decision to 
their corresponding cluster head. Finally, the cluster heads receive messages from their sensor nodes and remember their corresponding nodes. For each cluster, the corresponding cluster head chooses a set of $\mathrm{m}$ associates, based on signal analysis. Energy consumption of each node during data transfer varies with respect to the distance from their respective head sets and head set to the base station via other nodes involved in the network. When the clusters are being formed by the network, head sets are also allocated to decide the optimal number of

clusters. Cluster is being optimised based on the energy level consumption in the network [10]. Head set size and energy consumption are directly proportional to each other; such that the head set size optimisation in turn decides the power consumption of the network. Once the cluster is being decided with their respective head sets then the source and destination is being decided from the base station. The network is being monitored from the base station to have entire control over it. The "hello packet" is sent from the source to the destination by means of partial flooding using the right hand rule [8]. The flooded packet is being tracked by the base station to form the routing table, to decide the optimal route with respect to energy consumption, shortest path and less delay. The optimal route decision is based on the shortest delay path and less energy consumption in the network as shown in flowchart represented in Fig.2. The routing table is used to decide the path for the transmission of data in the network. The processed information reaches the base station where the signal is being efficiently used to monitor the physical changes in the environment.

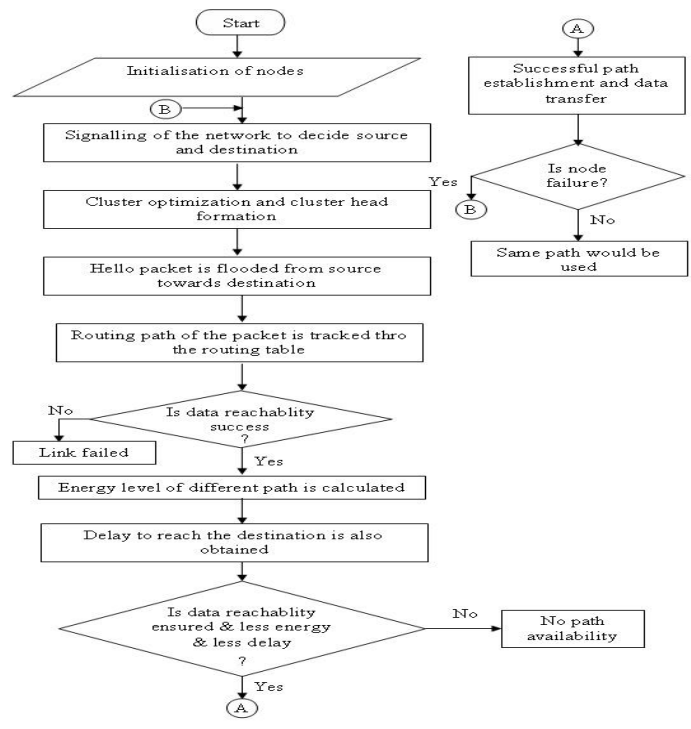

Fig. 2 Flowchart of the modified algorithm

\section{RESUlTS AND Discussion}

A WSN is simulated using ns-2 with 1000 nodes and 500 nodes. The nodes dynamically form a group of clusters and the cluster information is stored in their respective cluster heads. The source node and destination node are assumed. The source node transmits the "hello packet" to all neighbourhood nodes through right hand flooding technique. The flooded packets are tracked and a routing table is formed at the base station. Different path to deliver the packets is found, data reachability is ensured and a routing table is formed with all the successful routes. Once the routing table is formed, the optimal route is selected based on packet delivery delay, less energy consumption and number of hops.

Fig.3 illustrates the variation in optimum number of clusters with respect to the head-set size variation from 1 to 10 with 1000 nodes. As the graph shows, the head-set size cannot be greater than 6 because more number of head-set would lead to more number of clusters, which in turn consumes more power in the network. The head set size is fixed to 6 because of the consideration of both power and time taken for a packet to reach the destination. The time factor is being calculated using number of hops and delay factor of each sensor node. Delay factor is the time taken for a node to transmit data to the adjacent node. The maximum number of clusters and the head-set are the two major factors which decide the optimal route.

Fig.4 demonstrates the energy consumption with respect to the number of clusters. As expected, the energy consumption is reduced when the number of clusters is optimised. Moreover, the energy consumption is lower when head-set size is 3 as compared to head-set size of 1 because if the head-set is being 1 then the total network is being with a single cluster head will consume more energy to transmit signal to the base station. In case if the head-set size increases then the energy consumption decreases as shown in the graph. Thereby, even if the head-set size keeps on increasing more than the optimised value energy consumption in the network would be high. Therefore optimisation of head-set size and the number of clusters formed is also necessary for the energy efficiency in the network.

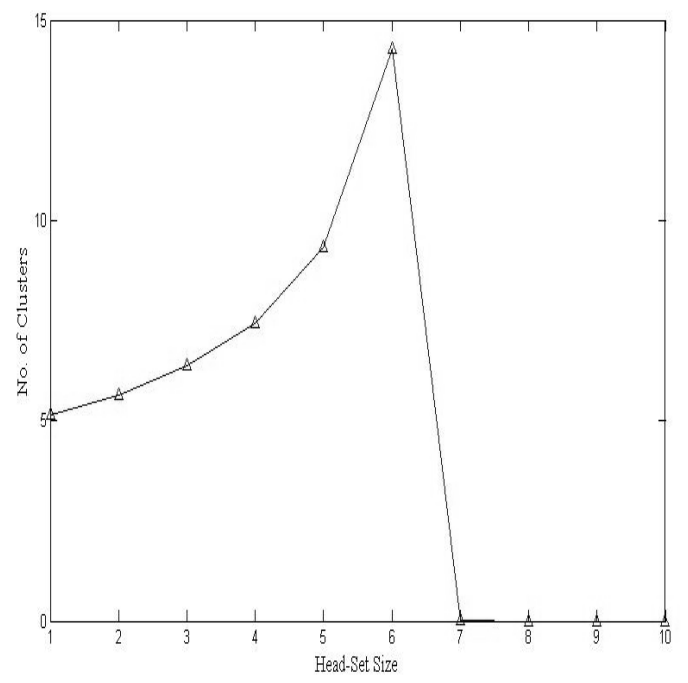

Fig. 3 Optimum number of clusters for 1000 nodes

Fig. 5 shows the variation in the energy consumed per node with respect to the number of clusters and network diameter. The figure also shows that energy consumption is reduced when the head-set size is increased. From the figure, it is implied that for a network which consists of 1000 nodes, the optimum range of clusters lies between 1 and 20. It is inferred, as the number of cluster increases the energy consumption decreases. 
Fig.6 illustrates the energy consumption for transmitting hello packet in different routes with respect to the number of clusters. It can be seen that the energy consumption values for different routes is less as number of cluster increases. If the cluster size increases the energy consumed in the network for transmission would be decreased but the need for aggregation node increases. If the aggregation node increases the power consumed by aggregation node would be high. This will lead to power drain of the aggregation node and failure of nodes in a short span of time. Therefore, cluster size should not be too low or too high and is optimised as 6 .

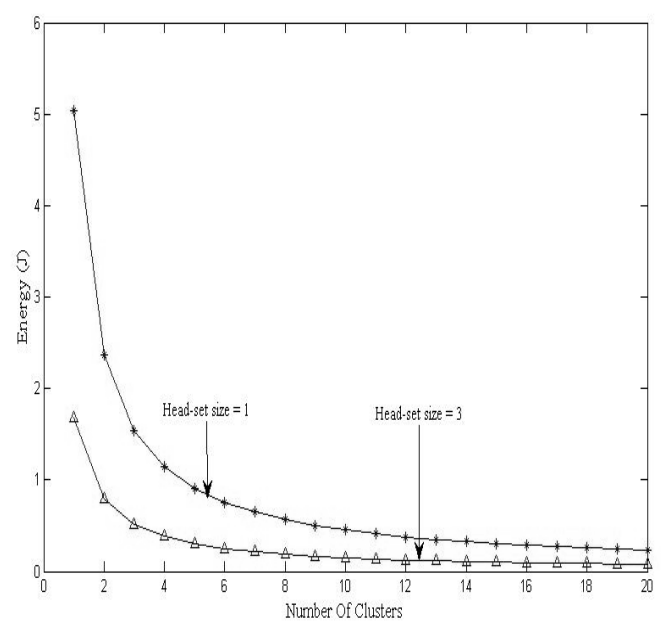

Fig. 4 Maximum number of clusters

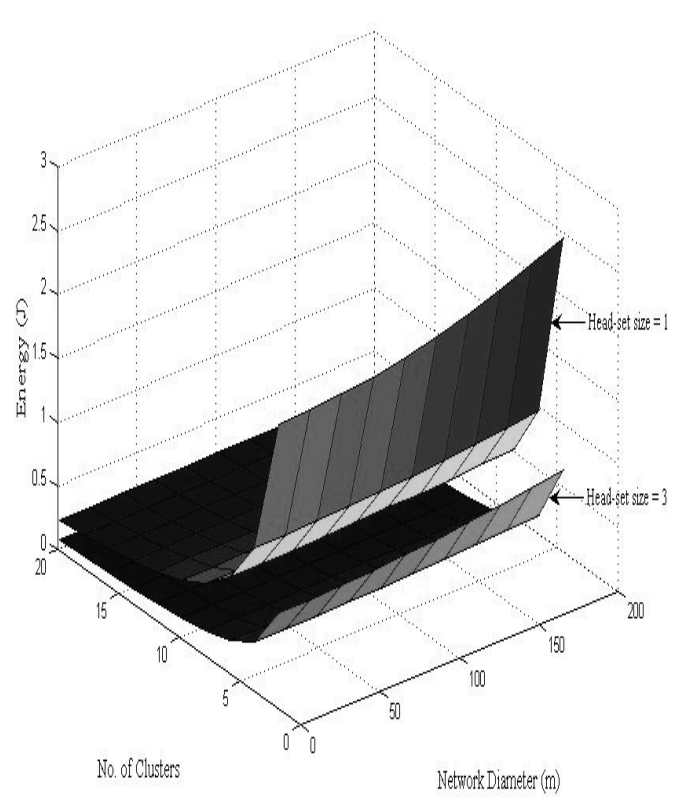

Fig. 5 Coverage, cluster and energy optimisation

A network should transmit the data successfully and quickly with less energy consumption. Once successful delivery and energy optimization is obtained, packet delivery delay (PDD) can be evaluated. A hello packet is transmitted through the network and the time taken to reach the destination is identified. Fig.7 shows the transmission time for different routes. These routes are compared with the energy optimised routes and optimal route is selected based on both energy consumption and delay. Fig. 8 reveals the time consumption for transmitting a sample data through first five optimal routes in a network which consists of 1000 nodes. The data is transferred only through the optimal route. In case if the optimal route becomes congested then the data is transferred through the next optimal route which is already identified.

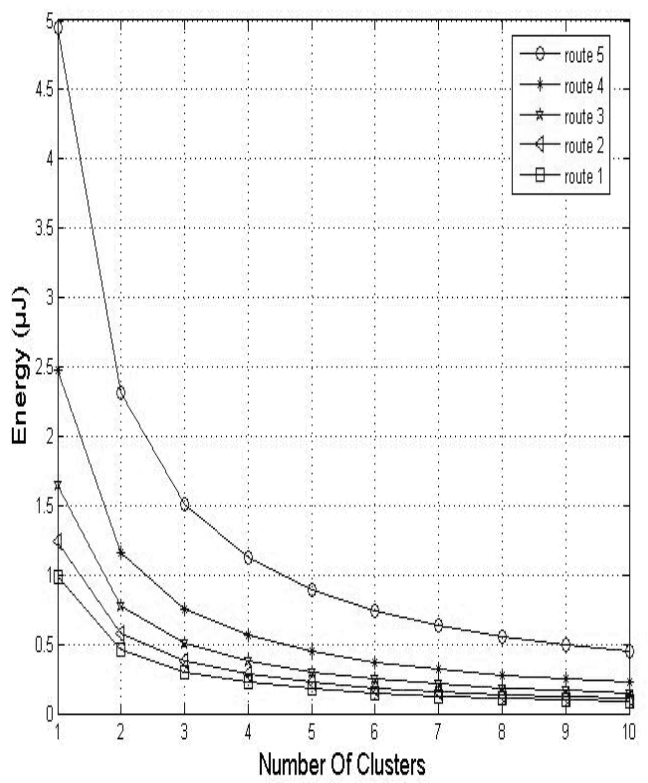

Fig. 6 Energy consumption for hello packet through 1000 nodes

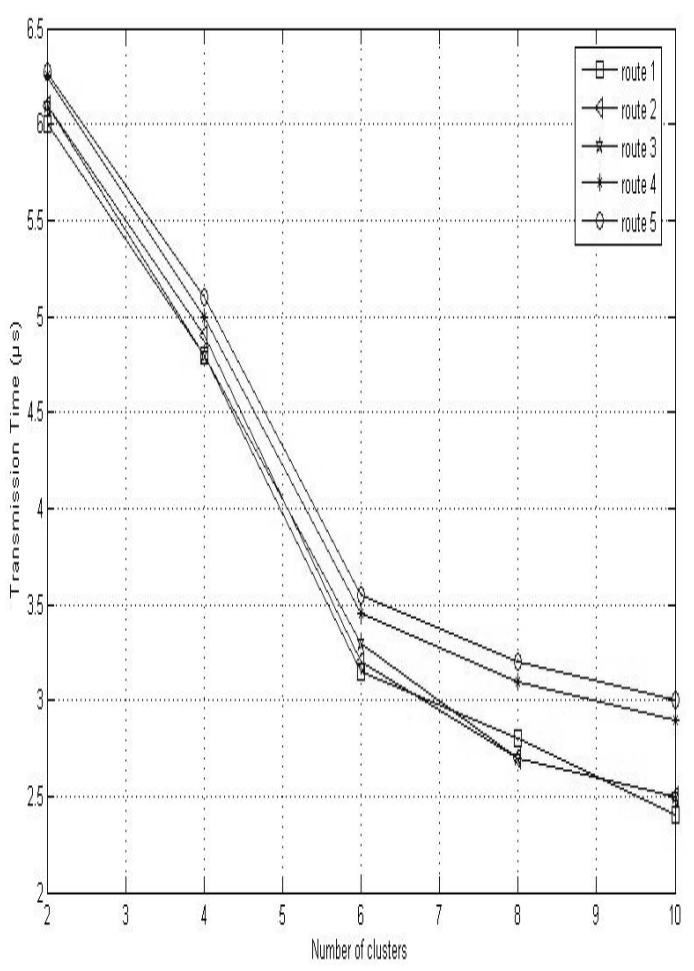

Fig. 7 PDD for hello packet 


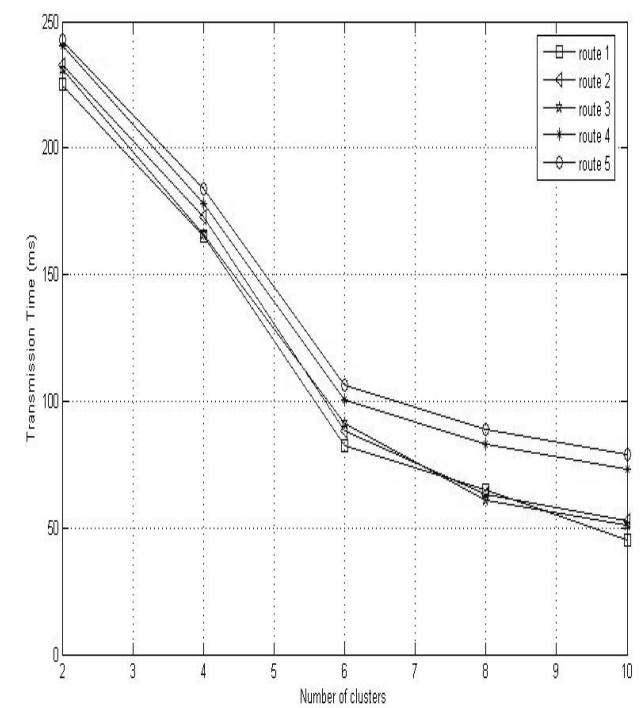

Fig. 8 PDD for 50 packets of sample data in 1000 nodes

\section{CONCLUSION}

The modified GPSR routing protocol strives to address the unique requirements for sensor network applications. It provides a robust, energy-efficient routing protocol with the ability to route messages from node to node and guarantees the delivery of packets under situations where non-uniform transmission ranges exist. A geographical routing protocol was developed and implemented for successful data delivery to any destination within the network or to the base station. The results of proposed optimal routing indicate that the energy consumption can be systematically decreased by optimizing the clusters and head set size which also increases the efficiency of the network. The energy efficiency of the network is increased by ensuring the data reachability using proper routing technique. If the path is being established from source to destination, the particular path might get loaded due to the traffic conditions. If traffic density is more, then an alternative path needs to be selected from the routing table with optimal energy conservation and shortest path with less traffic. This selection of path will further improve the routing technique by reducing the PDD and energy consumption. The PDD and scalability of the network increases the efficiency of WSN. This consideration proves this routing technique as an efficient routing protocol for WSN.

\section{REFERENCES}

[1] Jian Chen, Yong Guan and Udo Pooch, "Customizing a Geographical Routing Protocol for Wireless Sensor Networks", Proceedings of IEEE International Conference on Information Technology: Coding and Computing, Las Vegas, Vol.2, pp.586-591, April 2005.

[2] C. E. Perkins and E. M. Royer, "Ad hoc On-demand Distance Vector Routing", Proceedings of IEEE International Conference on Mobile Computing System and Applications Workshop, New Orleans, pp.90100, February 1999.

[3] W. Heinzelman, A. Chandrakasan and H. Balakrishnan, "EnergyEfficient Communication Protocol for Wireless Microsensor Networks", Proceedings of IEEE 33rd Hawaiian International Conference on System Sciences, Maui, Vol.10, pp.1-10, January 2000.

[4] Young-Bae Ko and Nitin Vaidya, "Location-aided routing (LAR) in Mobile Ad Hoc Networks", Proceedings of ACM/IEEE 4th Annual International Conference on Mobile Computing and Networking, Dallas, pp.66-75, October 1998.

[5] Shijin Dai, Xiarong Jing and Lemin Li, "Research and Analysis on Routing Protocols for Wireless Sensor Networks", Proceedings of
IEEE International Conference on Communications, Circuits and System, Hong Kong, Vol.1, pp.407-411, May 2005.

[6] Arjan Durresi, Vamsi Paruchuri and Leonard Barolli "Delay-energy aware routing protocol for sensor and actor networks", Proceedings of IEEE 11th International Conference on Parallel and Distributed Systems, Fuduoka, Japan, Vol.1, pp.292-298, July 2005.

[7] Mario Strasser, Andreas Meier, Koen Langendoen and Philipp Blum, "DWARF: Delay-aware Robust Forwarding for Energy-Constrained Wireless Sensor Networks", Proceedings of the 3rd IEEE International Conference on Distributed Computing in Sensor Systems, Santa Fe, pp.64-81,June 2007.

[8] B. Karp and H. Kung, "Greedy Perimeter Stateless Routing," Proceedings of the 6th Annual International Conference on Mobile Computing and Networking, Boston, pp.243-254, August 2000.

[9] Min-Gu Lee and Sunggu Lee, "Data Dissemination for Wireless Sensor Networks", Proceedings of IEEE 10th International Symposium on Object and Component Oriented Real-Time Distributed Computing, Santorini Island, pp. 172-180, May 2007.

[10] Peng-Jun Wan and Chih-Wei Yi, "Coverage by Randomly Deployed Wireless Sensor Networks", IEEE Transactions on Information Theory, Vol.52, no.6, pp.2658- 2669, June 2006.

[11] Wen-Jiunn Liu and Kai-Ten Feng, "Greedy Anti-Void Routing Protocol for Wireless Sensor Networks", IEEE Communications Letters, Vol.11, no.7, pp.562-564, July 2007.

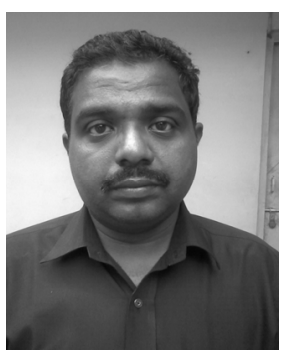

L.Nithyanandan received Bachelor of Engineering from University of Madras in 1992, Master of Technology in 1999 and Ph.D. degree in 2006 from Pondicherry University. $\mathrm{He}$ is working as an Assistant Professor of Electronics and Communication Engineering, Pondicherry Engineering College, Pondicherry, India. $\mathrm{He}$ is a gold medalist in PG and has been awarded with chief minister medal for his outstanding performance in PG. He has more than 15 publications in National / International conferences and Journals. His areas of interest include Sensor Networks, Telemedicine, Spread Spectrum Techniques and Wireless Communication

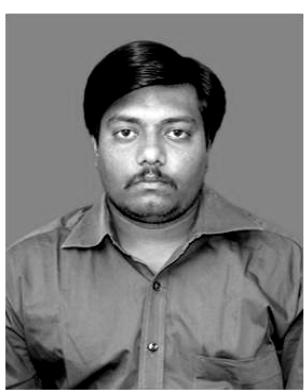

G.Sivarajesh received Bachelor of Engineering from University of Madras in 2004, Post Graduate Diploma in Human Resource Management in 2006 and Master of Technology in 2008 from Pondicherry University. He has been awarded top grade during his project course work in his PG studies. He has worked for HP as Senior Technical Support for 2 years. His areas of interest include Sensor Networks, Computer Networking and Wireless Communication

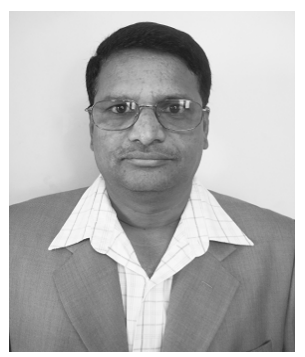

P. Dananjayan received Bachelor of Science from University of Madras in 1979, Bachelor of Technology in 1982 and Master of Engineering in 1984 from the Madras Institute of Technology, Chennai and Ph.D. degree from Anna University, Chennai in 1998. He is working as Professor in the Department of Electronics and Communication Engineering, Pondicherry Engineering College, Pondicherry, India. He is also a visiting professor to AIT, Bangkok. $\mathrm{He}$ has more than 80 publications in National and International

Journals. He has also presented more than 150 papers in National and International Conferences. He has guided $7 \mathrm{Ph} . \mathrm{D}$ candidates and is currently guiding $8 \mathrm{Ph} . \mathrm{D}$ students. His research interests include spread spectrum techniques and wireless communication, wireless adhoc and sensor networks. 